\title{
General Collective Intelligence as the Emerging Paradigm in Human-Centric Design for Sustainability
}

\begin{abstract}
Andy E. Willliams ${ }^{1}$

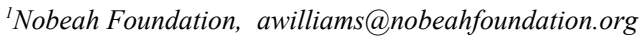

Abstract

A recently developed framework for modelling cognition defines General Collective Intelligence or GCI as Collective Intelligence $(\mathrm{CI})$ with general problem solving ability. Where CI uses the intelligence of crowds to optimize decisionmaking, a GCI must also optimize the choice of problem to solve. This framework represents a GCI as an adaptive problem solving system with problem solving segmented across a hierarchy of problem solving domains, one of which is adaptation through cooperation between functional components of the system. This domain defines how functionality is segmented across different components of a system in order to maximize outcomes, or in summary, balances centralization with decentralization. Where one function is more important to overall fitness than another, centralized cooperation prioritizes that function so that overall outcomes can be maximized. Decentralized cooperation maximizes outcomes for all participating components equally to remove the barriers that align decision-making with the interests of a subset of the group, which forces groups to solve the wrong problems. One such group problem is design and manufacturing for sustainability. Recent work has challenged the idea that process improvements will yield technologies with enough of an increase in efficiency to permit green growth while still reducing climate and other environmental impact. This paper proposes that designing all products and services according to the principles of GCI gives sufficient competitive advantage to businesses that cooperate to reduce consumption and increase sustainability, to make green growth not only possible but reliably achievable. This paper also provides an overview of what GCI based design and manufacturing of products and services for sustainability looks like. Leveraging GCI to achieve sustainability is explored as an example of biomimicry, and nature is shown to use the same approach to design living things. From this perspective, organisms are a collection of cells that cooperate to optimize functional designs according to well-defined principles in order to maximize the sustainability of the organism as a collective. Sustainability is represented as a mathematical pattern of stability implemented through these principles, a pattern which the 3.5 billion year history of the earth has thoroughly tested, and which therefore is robust enough to be replicated in all products and services, and once launched is stable enough as a pattern of cooperation to be sustainable in all organizations.
\end{abstract}

Keywords: General Collective Intelligence, GCI, Human-centric Design, Sustainability

\section{Introduction}

From the functional modelling perspective taken in this paper, life functions as an adaptive problem solving process, a process by which living entities solve problems posed by their external or internal environments as required to achieve a sustained pattern of mathematical stability in their fitness to execute all of their functions. Since solving problems through physical reconfiguration of a system is design, and since stability in fitness is sustainability, life is the process through which nature achieves sustainability in design. Where human design processes reliably fail to achieve sustainability, and by the reasoning in this paper currently might not even be capable of sustainability, nature's design processes reliably achieve sustainability. Replicating nature's sustainable design process involves understanding how nature decides what problem to solve, what functionality to use to solve the problem, what components to spread that functionality over, and what functionality to put in each specific component.

But before undertaking the effort to replicate such a complex and abstract entity as "nature's design process", it's important to provide sufficient motivation. Here it is useful to clarify why the capabilities of nature's design process are unique and significantly more advanced than current human design processes. In summary, natures' design processes are capable of scaling to be orders of magnitude more massive, being capable of individually redesigning every cell, every component consisting of groups of cells, and every subsystem consisting of groups of components, in a multicellular organism potentially consisting of trillions of cells, as well as being capable of redesigning the entire organism as a whole. From 
designing the organic molecules that give spider silk it's incredibly high tensile strength, to designing human consciousness, nature's design processes are reliably capable of designing functions that require Nth order interactions between components, where $\mathrm{N}$ is too high for current human design processes to reliably discover such complexity.

General Collective Intelligence (GCI) introduces functionality that changes this. A GCI orchestrated group design process might have the capacity to enable billions of people to suggest a set of functions that together might solve a targeted problem. Or to enable those people to suggest an alternate problem that might be targeted. The GCI orchestrated group design process might then enable those people to suggest designs that implement those functions, while also suggesting variations of the designs for each component so the group could collectively explore different configurations. The GCI orchestrated group design process might also systematically explore all available patterns of cooperation by which outcomes can be increased, and explore all available chains of cooperation incorporating those patterns. By systematically exploring the possible design configurations by which a collective outcome might be achieved, and by systematically exploring all possible chains of cooperation by which the probability of achieving that outcome and the scale of that outcome can be maximized, it may be possible to reliably arrive at a solution that increases collective outcomes to the point that cooperating to implement that solution provides sufficiently powerful advantage for the group to do so. Furthermore, reliably converging on selecting such a design as a group may be reliably achievable even where that solution is more complex than current design processes are reliably capable of. And finally, by incorporating adaptive design processes into the design process itself, that increase in value can reliably become self-sustaining.

\section{Methods}

Through identifying the functionality defined by a recently developed Functional Modelling Framework (FMF) as being required for a group decision-making system satisfying the criteria of a GCI within that framework, the impact of that functionality on sustainability, and on the design approaches necessary for sustainability, were assessed.

\section{Human-Centric Functional Modelling}

From a human-centric functional modelling perspective, entities can be defined as consisting of functional components, each of which executes a set of functions. These functional models are human-centric in that the functions can be directly perceived within innate human awareness rather than being deduced according to theoretical models. This functional modelling approach provides experientially verifiable definitions of terms that when defined intellectually are ambiguous, which allows groups to collaborate on problems of far greater complexity. In other words, intellectual reasoning has a capacity to arrive at truth that is finite (limited to cases in which adequate reasoning and the facts to plug into that reasoning exist) and potentially unreliable (reliable only where such reasoning is simple enough to be accurately computed). Experiential reasoning has a capacity to arrive at truth that is infinite (the truth of an infinite number of observations can be experienced) and reliable (experience can reliably be observed wherever an observation can be accurately identified as one's experience). The more experiential and less intellectual the discussion of a system is, potentially the more capable that discussion is of reliably converging on the truth.

Entities can then be represented as transforming themselves through all the states accessible through their functions, so that they move through a functional state space. This definition allows living entities to be distinguished very simply in terms of sustainability. Non-living entities such as rocks may change state (for example heat up during the day and cool down at night), but such changes are deterministic with respect to environmental forces, and therefore sustainability in such entities requires only stability in the dynamics of the environment. For the purposes of this paper we will ignore this sustainability as being outside of human control. However sustainability in a non-deterministic entity such as a living organism, or such as a 
city, requires stability in the entity's internal dynamics, which can be very simply expressed in terms of such a functional model.

\section{Defining Sustainability}

As mentioned, an organism, a system of organisms, or any other living entity interacts with its environment in a nondeterministic way. From the functional modelling standpoint, such an entity navigates its functional state space, and in doing so navigates its environment through the interactions of those functions with that environment. This functional state space defines the domain within which the entity can adapt to it's environment (the entity's domain of adaptability). Sustainability in such an entity in a given environment is the ability of the entity to perform its functions in a manner that sustains this navigation, which requires the entity or its environment recreating inputs required by all these functions in a sufficiently shorter time frame than it takes for the entity to consume them, and requires the entity or its environment consuming waste outputs created by all these functions in a sufficiently shorter time than it takes to produce them, so that the entity's level of consumption and waste production can be maintained within the levels of those inputs and outputs in the environment that permit the continued function of that entity.

The domain of adaptability of the "change engine" model of General Collective Intelligence discussed in this paper is adaptation through collective reasoning. Sustainability is the collective's stability in selecting which of its collective reasoning processes to execute. Defining a three dimensional "fitness space" representing the system's fitness to execute all its functions, this stability is achieved by defining an algorithm designed to constrain the system's dynamics in that fitness space so they follow a stable attractor governed by the Lorenz equations for convection, even though the selected sequence of reasoning processes itself may potentially be unstable and chaotic (Williams, 2020e).

\section{Defining Collective Intelligence and General Collective Intelligence}

From the functional modelling perspective human-like cognition navigates a functional state space containing the concepts available to the system (a conceptual space). From this functional modelling perspective solving a specific problem equates to finding a chain of reasoning that creates a path from one set of concepts representing one point in conceptual space, to another point in conceptual space. General problem solving ability equates to the ability to sustain navigation through the entire conceptual space so that it is potentially possible to navigate from any problem to any solution. Having the ability to sustainably navigate the entire conceptual space (having general problem solving ability) equates to the cognitive system having the capacity to define which problems to solve and what reasoning processes to use to solve them.

From this perspective Collective Intelligence (CI) is a collective reasoning process analogous to narrow AI in that it solves a specific problem. Collective cognition (general collective intelligence) is a group process that is analogous to Artificial General Intelligence (AGI) in that it has general problem solving ability, including a broader ability to decide which problem to solve. This problem solving ability is collective in that is intrinsically aligned with the well-being of the group, as opposed to the intelligence of individual members of the group, which is intrinsically aligned with their individual interests (Willliams, 2020d). 


\section{Decision-Making without GCI may be Constrained to Solve the Wrong Problems}

Many in sustainable development have pointed out the difficulties that arise from identifying the wrong problem (Cockerill et al., 2017),(Cartledge et al., 2009). But the theory behind this model of GCI suggests that without GCI groups will in many cases not only tend to solve the wrong problem, but also tend to be constrained to do so (Willliams, 2020d). To understand why, imagine there is a famine that has left you and your family starving in South Sudan. Somewhere in Brussels, a program manager at a large humanitarian organization is deciding on what aid to send in response. Before deciding to send that aid, the program manager might go over the organization's long list of policies and procedures, perhaps making sure that any food aid meets the recommended minimum daily allowances of nutrients, perhaps making sure it comes from an approved supplier, perhaps making sure it comes from factories that meet its labor standards, and so forth, before finally sending three days worth of food aid after that process is concluded. Rather than considering all of this, you in South Sudan on the other hand might have preferred to just take the money equivalent to that three days worth of aid. Anything big donor organizations do is expensive. So with that money you might have been able to simply buy a month's worth of cornmeal flour to make ugali (a type of cornmeal porridge) from a passing trader that same day. Ugali is largely starch, and might not meet the donor organization's daily nutrient requirements. But it is familiar to the primarily starch based diet in rural Africa where meat is harder to come by (Schönfeldt and Gibson Hall, 2012), and will protect your family from starvation.

Or you might have preferred to take the money for that three days worth of aid and simply travel with your family to stay with relatives in neighboring Kenya, where instead of eating for three days, you and your family might be fed and sheltered for the next six months. In this way both of these options might result in levels of food security that are an order of magnitude or more greater. This example is somewhat contrived and may not represent the actual policies of donor organizations or the actual possibilities open to victims of famine. But when trying to maximize outcomes, this hypothetical example shows the importance of decentralizing decision-making to put some control about decisions over interventions into the hands of those impacted by those outcomes. Because each of these people is solving a different optimization problem. The person in the famine is solving the problem of the famine. The program manager is solving the policies of his organization. Centralizing decision-making so that it is in the hands of one entity is one of the many ways groups can be constrained to solving the wrong problem.

\section{Decision-Making without GCI may be Constrained to Select the Wrong Solutions}

Take consumer electronics as an example. Assume that over the last two years a consumer bought at least half a dozen headsets for conference calls, as they kept breaking. Each time the consumer would have had to discard the entire headset even though only one part was broken, because the headset was essentially designed as one piece. If the headset were designed so that the jack was separate, the cord was separate, the speakers were separate, and all the other pieces were separate, and all of those parts were "human-centric" in being replaceable by the user without any specialized tools or expertise, the consumer would have been able to replace just that one part. Rather than lasting for a few months, the headphones instead might last 20 years. This value proposition could provide those headphones with an unbeatable competitive advantage to consumers. But no current business could survive by planning to sell no more than one set of headphones to each customer during the customer's lifetime. As described in this paper, competition may in this way force companies to either compete to drive consumption as unsustainably as possible through planned obsolescence, or be replaced by another entity that will drive unsustainable consumption in their place. Because of this force, companies might be constrained to select the wrong solutions in terms of sustainability, not just in design, but throughout the entire product or service life-cycle. 


\section{Economies without GCI might be Constrained to Consume Unsustainably}

Technology can provide competitive advantage. This competitive advantage can potentially be used to win more resources, to then buy more technology, in a cycle of increasing inequality. While disruptive new technologies might dramatically shift competitive advantage to new players, this concentration of resources may still tend towards increasing this cycle of inequality for some entity, even if not the same one (Williams, 2020f). Competition and inequality are not intrinsically bad. In the natural world they are essential to the natural selection that makes ecosystems more fit. The issue is that technology removes the limits to our consumption from being what our appetites can hold, or what we can physically store or carry, and therefore also removes the limits to inequality. Removing the limits to inequality potentially means that organizations might never be allowed to stop competing to unsustainably increase the consumption of their customers so they can acquire further resources, without the threat of being eliminated from the marketplace by others who will do so in their place. This technology driven inequality has been called the "technology gravity well". Although inequality trends differ considerably depending on the concept used, some studies do show the constant increase in the global GINI coefficient (a measure of inequality) over all human history for which data is available, which appears to support the existence of such systemic forces (Goda, 2016). Where these forces might drive for-profit organizations to compete to increase the consumption of their customers, in non-profit organizations similar forces might drive organizations to compete for donations, rather than cooperating to maximize any collective maximize impact.

Defining a closed system to be one with no external inputs or outputs, and defining all national or regional groups of individuals to be open systems since all of them trade, then only all groups of individuals globally form a closed system. The importance of this distinction is that GCI is a system for maximizing the sustainability of resource consumption and waste production by a group, and therefore, viewing collective outcomes as any purpose to which the consumption of resources and production of waste might be dedicated, GCI is a system that maximizes the sustainable achievement of collective outcomes per unit of resources.

Just like an individual can't reliably win any race against competitors they do not control without the ability to consciously control the degree to which they run their fastest, a group can't reliably achieve any collective outcome in an unconstrained environment without control over the degree to which it maximizes its ability to sustainably achieve that collective outcome per unit of resources. Since a great many strategies for maximizing collective outcomes are solely within the domain of GCI, a closed group cannot reliably achieve entire classes of sustained collective outcomes without GCI. Therefore any ability of any current decision-making systems other than GCI to reliably achieve such collective outcomes, such as potentially including eliminating poverty, or reducing greenhouse gases, must reliably come at the expense of exporting the problem to other groups with which it is connected. From this perspective, the wealth of western economies might be connected with the poverty of the less developed countries that sell raw materials to the west and purchase high value-added products back in return. And the achievement of global greenhouse gas targets might be connected with the less developed nations capping their development at far lower levels of consumption than the west currently has.

Assuming GCI is a system for maximizing the sustainability of resource consumption and waste production by a group, and assuming sustainability is not reliably achievable in a closed technology possessing group without GCI for some specific class of outcome, then the lack of such a system essentially chooses the only remaining path to sustainability. Because without a system for maximizing sustainability of resource consumption and waste production, the only way to reliably avoid increasing consumption and waste production past any "global tipping points" might be to continually decrease the size of the group doing the consumption and waste production. However, such action requires a degree of centralization of 
authority that might itself be dangerous to sustainability. Because the only ways to achieve control of that centralized authority (aside from conflict) might again be to acquire resources through winning the competition to encourage the most unsustainable consumption of products and services possible. And participants may be locked into this competition until a clear winner emerges.

\section{General Problem Solving Ability as an Adaptive Process}

From the functional modelling perspective the functional components that make up living organisms consist of a hierarchy of adaptive processes, each of which can be decomposed into a set of functions which operate in that adaptive domain. All living organisms have the potential capacity to adapt to any state of their environments that can be accommodated within these domains of adaptability. In this sense all living organisms have general problem solving ability. The challenge in achieving sustainability is to increase the size of the total composite domain of adaptability so that sufficient functions to achieve that sustainability in a given environment are available.

Human beings are the one species known to have the capacity for abstract reason as an adaptive domain. In order to have general problem solving ability in the reasoning domain, and therefore to have the capacity to solve a wide range of problems of individual sustainability, human beings require a general problem to solve. That is, a general problem which all problems in the cognitive system can be defined as belonging to. And the one general problem shared by all humans, is the problem of achieving "well-being". Adopting the most general possible definable problem of well-being, which is proposed here to be fitness to execute all the individual's functions, enables the organism to adapt to solve any problem that impacts fitness in performing any function, even functions that adaptive processes such as evolution may not have created yet. In the same way, solving such a general problem of well-being might also enable the organism to eliminate functions that evolution or other adaptive processes no longer see as necessary.

Where this functional modelling perspective suggests that an innate metric for individual well-being is a required component of individual reasoning as an adaptive domain, lacking such a common definition for collective well-being (as would be provided by a GCI), groups cannot have the capacity for collective reasoning as an adaptive domain, and without collective reason (as would be provided by a GCI as well), groups cannot have the collective general problem solving ability that might be required to solve a number of problems of general sustainability. Where current sustainability initiatives might lack a definition of collective well-being, and therefore lack a general problem to solve, the model of collective cognition (GCI) described in this paper aims to provide the capacity for collective general problem solving ability through providing a quantifiable definition of collective well-being and a mechanism for collective reasoning to achieve it.

\section{Results and Discussion}

A group decision-making system satisfying the criteria of a General Collective Intelligence was found to be necessary in design and other processes for sustainability to be reliably achievable.

\section{The Implications of Collective General Problem Solving Ability on Collective Challenges}

The first implication of GCI on collective problem solving ability is that the conceptual space is open and can therefore potentially include any definition of a problem or any concept of any solution within the limits of complexity representable 
by the system. So in this sense a GCI has the potential to target any collective problem that exists within its conceptual space, and therefore that it can conceive.

However, as mentioned, any cognitive system might be limited in the level of complexity of reasoning it might be able to use to navigate from one point in its conceptual space (the problem) to another point in that space (the solution), since some solutions (i.e. connecting some points within the conceptual space) might require navigating well outside the conceptual space available to it. Or might require navigating to points within the available conceptual space that are too fine to be resolved. For a GCI to have significantly greater "intelligence" than any individual in the group, the GCI must have access to some portion of the conceptual space available to each individual in it, as well as some access to each individual's library of reasoning processes, so the collective conceptual space and collective ability to navigate that space (the group's collective "intelligence") might be many orders of magnitude greater than that of any individual. For example, where an individual, or a group without GCI, might have the capacity to reason through evaluation and comparison of a few dozen proposed solutions, a GCI forming such a collective super-intelligence might be able to compare billions.

The second implication of GCI is that it can potentially remove the barriers to massive cooperation, and use that cooperation to increase the probability of impact on collective challenges and the potential magnitude of that impact, as well as increasing the value of that cooperation to the point that impact becomes sustainably self-funding so it can be deployed at massive scale. Case studies exploring the deployment of entire chains of businesses with only a few of the many available patterns of collectively intelligent cooperation show this potential to reliably provide powerful competitive advantage for groups of organizations that cooperate in this way to achieve the common good. Specifically, where the value of cooperation is greater than zero, collective intelligence can be used to remove the barriers to scaling cooperation so the value of that cooperation can be increased as much as needed to reliably create unbeatable competitive advantage for those businesses that cooperate towards the common good, in this case cooperating to reduce consumption. In the case of the head set, this means creating the capability to search for every opportunity for any business to gain from sharing a design component, making it possible to form entire value chains that cooperate to share designs so they might reuse the same part between them,. This shared design essentially subsidizes each specific component ("cross subsidization"). As the number of businesses in the cooperative value chain increases, so does the size of the subsidy it can cooperate to afford. The size of the value chain could feasibly be increased to undercut any competition, creating a potentially unbeatable competitive advantage that would help guarantee the sales to support the value chain (Williams, 2019). With this approach the total cost of the headset might be lowered, and the market share might be raised, to the point where a business could quite comfortably sell only one headset per lifetime. Design for collective intelligence is a methodology to help identify the opportunities to implement modularization and the other principles displayed by collectively intelligent products and services.

A cooperative value chain in which one participant gives an agreement to purchase from another participant in return for receiving an agreement from yet another participant to purchase from them, is an exchange of value that we have named an Nth-order barter transaction. Simple (first order) barter transactions in which one person exchanges something of equal value to another person are easy for people to negotiate. However, in an Nth order barter transaction the first person gives something to the second person, the second person gives something to the third person, and so forth until the Nth person give something to the first person. Even if all the participants were brought into a single arena, Nth order barter transactions might be too difficult for humans to detect. But for computers the task is simple. Such barter transactions can be among a network of peers (Nth order network barter transactions), or can be hierarchical (Nth-order hierarchical barter transaction). In an $\mathrm{N}$ th order hierarchical barter transaction $\mathrm{N}$ people each give something to 1 person in return for something of $\mathrm{N}$ times that value which they share. An example of this is a group of farmers who each give a road builder part of their harvest so they can all have a road to market. By being able to define these transactions or hybrids of these transactions algorithmically, a cooperative platform can find these transactions and lower the barrier to any outcome that can be facilitated by a product or service, even where these transactions are far too difficult for any human being to identify unaided. 
Cost sharing in a hierarchical barter transaction enables each participant to contribute value in return for something of equivalent cumulative value whose usage they share. But in an Nth-order hierarchical cost sharing transaction, in addition to some value being distributed by one party between $\mathrm{N}$ other parties in return for the value they each contribute, there is also an outcome that is multiplied by some factor $\mathrm{M}$, where $\mathrm{M}$ is the ratio between the magnitude of impact caused by the $\mathrm{N}$ parties versus the magnitude of impact caused by only one party. Given that M describes the multiplication of outcome per unit cost, it also describes the cost savings factor per unit of outcome. An Nth order network barter transaction in which $\mathrm{M}$ participants contribute to a common outcome might achieve the same multiplication in impact. In both cases, identifying cooperation as Nth order barter transactions in which value is exchanged, and identifying Nth-order sharing as an Mth-order cost reduction transactions, allows opportunities for cooperation and cooperation to be more objectively understood.

modelling cooperation and sharing in this way enables these opportunities to be manually defined, or manually detected more easily. When semantic searches become available so that complex entities like businesses can be understood by computers the same way humans understand them, modelling cooperation and sharing in this way will allow such opportunities to be searched for and found algorithmically where currently they are too complex for human beings to detect. The capability to detect the maximum opportunities for cooperation and cost-sharing also maximize the degree to which cooperation and sharing can support a given group of businesses.

In summary, within it's domain of adaptability a GCI can potentially increase impact on collective challenges until impact is reliably achievable. These challenges include sustainability. Because of it's potential capacity to drive transformative change the model of GCI discussed in this paper has been called the "change engine".

\section{Using GCI to Increase Impact on Sustainability Until it is Reliably Achievable}

By the arguments provided earlier in this paper, for every product or service that involves group processes anywhere in its life-cycle, from design to recycling, the principles of GCI can potentially be used to target a significantly increase impact in sustainability.

Collectively intelligent product or service development aims to create the ability to reliably explore all of the currently available solution space and to reliably select the best available solution in that space so that developing solutions which facilitate a significantly improved impact on targeted outcomes as compared to other development processes is reliably achievable. Collectively intelligent design aims to create the ability to reliably explore all currently accessible design configuration space and to reliably select the best available design in that space. It also aims to create the ability to reliably redefine design problems in a sufficient number of ways to explore all currently known outcomes that can be targeted so that all available categories of designs capable of targeting those outcomes can be explored.

Where conventional development has known cognitive or other biases that may prevent certain categories of solutions from being selected even where optimal, collectively intelligent development aims to create the capacity to methodically compare all known candidates for all known components of that solution and to select the best for each, so that development reliably converges on the optimal overall solution. Where conventional design processes share similar constraints, collectively intelligent design aims to create the capacity to reliably design solutions that facilitate a significantly improved impact on targeted outcomes, or to design components which redefine problems addressed by multiple solutions so they can be 
addressed by that design, and therefore to design components that can facilitate even greater improvements in impact, as compared to other design approaches.

In development or design processes without GCI, the space of problems to be solved through design, and the space of designs proposed to solve those problems, are suggested to both not only be constrained, but to be constrained so that the most sustainable definitions of the problem and the most sustainable solutions are likely not included. In fact, as discussed in this paper, these constraints might prevent sustainability from being reliably achievable globally at all without GCI.

A case study for the potential application of GCI to the development and deployment of renewable energy solutions (the proposed Collective Intelligence Based Renewable Energy Program (Williiams, 2020b)) suggests that collective intelligence has the potential to make a far bigger impact on greenhouse gas emissions than carbon taxes or any other specific approach, since current decision-making processes are proposed to be driven by perverse incentives that might actually prevent climate change from being reliably addressable. Similarly a case study for the potential application of GCI to the design and deployment of smart sustainable housing (the proposed Design for Change Nairobi Challenge (Williams, 2020c)) suggests that collective intelligence has the potential to make a far bigger impact on sustainable or "green" growth than any current sustainable development program, again, because current non GCI based sustainable development programs are proposed to carry perverse incentives that again might actually prevent their targeted outcome from being reliably achievable.

The proposed Collective Intelligence Based Renewable Energy Program aims to leverage human-centric functional modelling to provide groups with a common model of the problem being solved, and it aims to use collectively intelligent cooperation in developing a solution that can reliably achieve a significantly lower cost of access to sustainable renewable energy, or achieve a significant increase in environmental sustainability of that renewable energy, as defined by metrics including carbon emissions (Williams, 2020b). The proposed Design for Change Nairobi Challenge aims to use a GCI platform (the Collaborative Design Platform) to engage a massive collaborative effort in designing a housing structure with the capacity to reliably lower the cost of living and increase earning capacity of occupants by a sufficient amount per month to lift families in the slums of Nairobi out of poverty. Targeting more than one million participants as designers, this challenge aims to leverage collectively intelligent cooperation to distribute the effort of exploring and comparing the benefits of integrating solutions from other projects, including renewable energy, aquaponics, or other solutions, so that it is reliably possible to achieve this increase in impact on poverty reduction (Willliams, 2020c). After this challenge has been completed in Nairobi, the same technologies and solutions are intended to be adapted to launch Design for Change challenges in Europe and North America. For example, the Design for Change Europe Challenge aims to use collectively intelligent value chains to build smart housing with the capacity to revitalize the economies of ghost cities by turning them into smart cities that generate massive job creation while attracting massive investment.

\section{Human-Centric Functional modelling as a Requirement for GCI Based Design and Other Processes}

Functional modelling is a necessity in exposing group processes to GCI. Just as an individual must make a cognitive model of their body, as well as of the tools and other objects they interact with, in a GCI the functions of the people in the collective, as well as the functions of the products and services at their disposal, are modeled so the representations of those functions are available to be manipulated by all collective design, manufacturing, and other group processes. The only representation of functional models that can be common to all individuals is one that is human-centric in the sense that it relies solely on our innate conscious awareness and cognition, rather than relying on accepting the validity of any implementation approach or theoretical framework.

Having defined this functional model for products and services, as well as the functional model for sustainability described earlier, GCI can be used to apply this sustainability model to every process in the entire life-cycle of every product or 
service. But most importantly, regardless of how good any system is at optimizing group outcomes, it can't be effective if it isn't used. Where rules to enforce any initiative can simply be broken, GCI adapts to adopt whatever intervention works best in each adaptive domain, so that group behavior can reliably converge both on using GCI and on achieving sustainability.

This human-centric functional modelling approach presented here attempts to formalize the way human beings intuitively create functional models of objects. Each function of a physical or virtual solution can be conceptually represented in terms of inputs, outputs, a context of execution, and an outcome related to that output, where for physical products that outcome is a physical transformation.

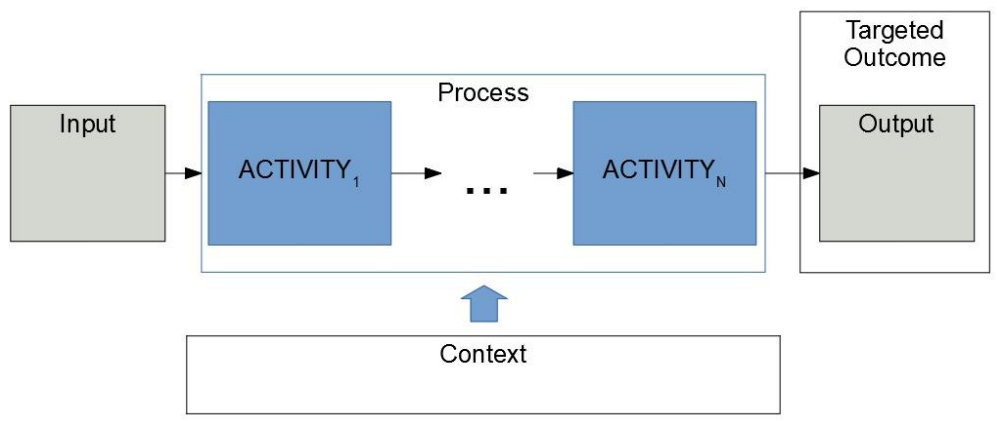

Figure 1: Functional model of a physical or virtual process.

Using a functional modelling approach, each functional component can be represented as having a set of functions through which it can change its state, such as by adding or removing other functional components. The set of states through which the functional component can transition are its functional state space. Interactions between functional components can similarly be represented in terms of a set of functions. These functions are in effect transforms that change the state of the functional components. These functions (transforms) map from one point in that functional state space to another. The composition of all the functional state spaces of all functional components then represents the functional state space of the entire assembly. Assembling a part then becomes a matter of defining a path through the state space of an initial functional component to an assembly of functional components representing the entire system. As an example, a screw that fastens a chair leg to the chair is a functional component of the chair. Turning is one of the functions of the screw. Through its function of turning, the screw transforms the functional state of the chair into an assembly consisting of these three functional components. That transition from the functional state of not having a leg, to the state of having a leg fastened by a screw, is a path through functional state space.

Of course, some functions that biological organisms perform are not repeatable and therefore not stable. For one-time functions repeatability can be only achieved through different instances of the organism. For example, an egg can be broken by its chick only once. But the function of being broken is repeatable across all eggs because chicks grow up to lay their own eggs. By contrast, an egg can be scrambled, but that isn't repeatable because no scrambled egg ever produced another egg. Being scrambled is not a repeatable function of the egg, its only a repeatable function of the person scrambling it. In the same way, a product or service can have functions that are not stable or repeatable. 
Defining the functional state space of a product or service as containing every stably occurring function it can perform, then representing every such function as a transform in that space, defines a single database of functions that can potentially enable all design and other efforts to collaborate in an automated way. While these principles have been manually implemented in actual projects (e.g. the "Smartwire" in the SmartGrid renewable energy project), automating these principles as cooperative design processes in a CAD platform as needed for massively collaborative design, requires further research.

\section{Introducing GCI into Design and Other Processes in the Life Cycle of Products and Services}

In living organisms, every functional component is methodically varied by the different adaptive processes (growth, reproduction, etc.). Those variants are tested for fitness across all of their functions, and the most fit designs are kept (survive). In physical products, this process can be replicated by defining functional components (e.g. television screen and computer monitor), and systematically varying the interfaces, and modularizing until the functional components have maximum fitness in executing the sets of functions required by all stakeholders (e.g. a television screen composed of multiple screen modules that can be replaced if one module cracks, where the modules can also be assembled into a computer screen, a smartphone screen, or other display).

In order to vary products and services so that the fitness of each variant across all potential chains of cooperation can be compared, functions of each functional component must first be identified using the functional modelling approach defined above so that all components with common functions can be found. The interfaces of these functional components must then be defined so those interfaces can be varied until they are potentially common between functional components. In the case of physical products this means varying the physical dimensions of the interface. For example, cars have different rim sizes and different tire sizes. Varying the rim sizes in increments between the maximum diameter, width, and other relevant dimensions, enables each configuration to be tested to find the one that maximizes fitness over different vehicle makes and models. In addition, introducing modularization, such as layers that can be stacked to make deeper rims, allows that variation to include parameters typical of other applications, such as motorcycle or bicycle tires. At some point, the competitive advantage of all these patterns of cooperation becomes too great for non-sustainable competitors to be viable. Whether it's at the point where sustainable cars cost $10 \%$ less than non-sustainable ones, or whether it's at the point where sustainable cars cost 10X less, at some level of competitive advantage that point must be crossed.

Fitness is defined here as capacity to execute all functions. In living organisms fitness goes from zero (no capacity to execute any functions, or in other words dead), to one (complete capacity to execute all functions). However, assessing the specific level of fitness of any system is the subject of ongoing research. Lacking equations for calculating fitness, such assessment will likely need to be made by assessing fitness in each given context for each category of system, and then simply using the fitness of that category as a starting estimate. For example, where the relative fitness of a new design of frictionless wind turbine has not yet been assessed, that wind turbine might be assigned to the category of frictionless turbines which may have $10 \%$ relative increase in fitness in generating electricity as compared to other wind turbines.

\section{Launching a GCI}

Leveraging the potential of GCI to target any collective challenge and to increase the value of cooperation until solutions become essentially self-funding, so that it is possible to deploy solutions at sufficient scale to significantly increase impact 
on collective challenges, a Collective Intelligence based Program to Accelerate the Sustainable Development Goals (CIPAASDGs) has been defined. Over it's first three phases, this program (which is still awaiting a feasibility study) aims to direct \$15 B USD towards sustainable development, including significantly increased deployment of clean renewable energy technologies, so that carbon emission targets become reliably achievable while maintaining economic prosperity. Over its entire ten phases, this initiative leverages collective intelligence with the aim of making its initiatives sustainably selffunding so that it's possible to reliably close the $\$ 23$ trillion SDGs funding gap identified by the UN. While this GCI infrastructure may be critical to achieving the SDGs globally, it may be difficult to justify any expenditure whatsoever to build it for any single project. In the same way that purchasing a backhoe cannot be justified for any single home building project. Splitting the cost across many different projects can lower the cost to the point where the benefits are great enough, and the cost is negligible enough, for the project to build a GCI to occur.

However, in addition to building it, some research (as mentioned in this paper) is also required to implement this very abstract collective intelligence infrastructure. This research is likely well outside the scope of what can be funded within any sustainable development program within the policies of any development agency.

In order to facilitate research to elaborate the elements of the model required by the CIPAA-SDGs program, a series of LSCORE (Large Scale international COllaborative REsearch) initiatives have been defined. The model of cognition discussed in this paper is a functional model independent of implementation. For this reason it is proposed to apply equally to individual cognition, systems of artificial cognition, as well as software organizing groups into a system of collective cognition (a GCI). By defining research projects in these different areas that each contribute to the understanding of the same underlying model, it is possible to use collectively intelligent cooperation between these projects to reliably incentive participation in them. Leveraging a common functional model of cognition that enables research from one initiative to be reused in another can significantly increase a participating researcher's impact. By significantly increasing the problems that each researchers work applies to, these initiatives have the potential to significantly increase the pool of stakeholders with an interest in the solutions, and therefore increase the funding available to each researcher.

However, if it is true that in the absence of GCI, public and private donor organizations may be driven more by incentives that centralize decision-making, then by the assumptions made in this paper, instead of driving such organizations to compete by continuously solving collective problems more and more efficiently, those incentives might in some cases effectively not be concerned with solving the problems at all. And if existing incentives are effectively unconcerned with solving the real problems, the challenge is then launching a GCI whose value proposition is significantly increasing impact, when an increase in impact doesn't in practice incentivize the organizations concerned. This reasoning creates a chicken and egg problem. Without GCI even charitable organizations might have a tendency to be constrained to compete to attain greater and greater control of philanthropic resources rather than be incentivized to create a GCI, regardless of the potential increase in outcomes. Surveys under way suggest that even conceptual case studies (Williams, 2020a) suggesting the potential for GCI to multiply effectiveness in solving collective challenges by up to seven hundred and fifty fold do not reliably incentivize all governments or donors to participate in studies of the feasibility of implementing one. For this reason, in the current centralized decision-making paradigm it may be difficult to create sufficient initial incentive for stakeholders to implement a general collective intelligence, regardless of whether doing so might be essential to actually solving the most critical social, environmental, economic, or other collective challenges globally.

The LSCORE and CIPAA-SDGs projects aim to make launch reliably achievable by using the principles of GCI to reduce the scope and cost of each sub-project, to increase the incentives to participation, and to decouple the projects so they can be funded independently, such that when sufficient mind share is created there might be enough stakeholders with an interest in doing whatever is projected to have the highest impact on collective global well-being, rather than having a cognitive bias towards or belief in a specific means for achieving it, for the projects to be reliably funded. In this way the principles of GCI are being used to remove the barriers to the launch of GCI being funded by a great many smaller contributions. On the other 
hand, according to these same principles the $\$ 40 \mathrm{M}$ cost of initiating all these projects together has also been defined to remove the barriers to a single stakeholder funding them.

\section{Conclusions}

As discussed in this paper, the reason why hasn't nature created unsustainable systems until human beings may be that competition in nature is limited in its ability to create inequality, and therefore organisms can't benefit from unbounded and therefore unsustainable consumption. How can human beings introduce organic limits to consumption? The answer suggested in this paper is through using GCI to create the capacity for greatly increased problem solving ability, and to create the capacity for massive cooperation, as well as the capacity for other adaptive processes that naturally converge on collective well-being, which includes sustainability. GCI aims to radically increase the size of the collective conceptual space, and to radically increase the speed at which the collective can navigate that space, to radically increase the group's general problem solving ability. Through increasing problem solving ability to the point that solutions lie within accessible parts of the collective conceptual space, collective problems such as sustainability might reliably be addressed in an ongoing way.

Nature has defined a robust model for sustainability that can be abstracted and generalized so it can be reused wherever it applies. Human-centric functional modelling of collective problems and prospective solutions creates the potential capability to reuse that model of sustainability in design and the rest of the entire life-cycle of every product or service. GCI creates the potential capacity to search for those opportunities and to orchestrate and reliably incentivize the cooperation required to take advantage of them. In introducing general problem solving ability to groups GCI vastly increases the size of the collective cognitive space, and makes new collective reasoning processes available to navigate that space, so that parts of the collective cognitive space containing optimal solutions to sustainability might be accessible where they may not have been before. If so, then even though it is an abstract concept, GCI might be more important to sustainability than any problem of sustainability itself.

\section{References}

Cartledge, K., C. Dürrwächter, V. Hernandez Jimenez, and N. P. Winder. 2009. Making sure you solve the right problem. Ecology and Society 14(2): r3. [online] URL: http://www.ecologyandsociety.org/vol14/iss2/resp3/

Cockerill K., Armstrong M., Richter J., Okie J.G. (2017) Integrating Science and Society for Environmental Realism. In: Environmental Realism. Palgrave Macmillan, Cham

Goda, Thomas. (2016). Global trends in relative and absolute income inequality. Ecos de Economía, 20(42), 46-69. https://dx.doi.org/10.17230/ecos.2015.42.3

Schönfeldt, H., \& Gibson Hall, N. (2012). Dietary protein quality and malnutrition in Africa. British Journal of Nutrition, 108(S2), S69-S76. doi:10.1017/S0007114512002553

The Collective Intelligence Based Program to Accelerate Achievement of the Sustainable Development Goals as a Case Study for Collectively Intelligent Program Design. SocArXiv, n.d. Web.

Williams, Andy E., 2019, The Relationship Between Collective Intelligence and One Model of General Collective Intelligence, Computational Collective Intelligence, 11th International Conference, ICCCI 2019, Hendaye, France, September 4-6, 2019, Proceedings, Part II, Pages 589-600

Williams, Andy E., 2020, a, A Proposed Collective Intelligence Based Renewable Energy Solution Development Program, working paper (2020).

Williams, Andy E., 2020, b, Collective Design Cognition in a Proposed Design for Change Nairobi Challenge, working paper (2020).

Williams, Andy E., 2020, c, General Collective Intelligence and the Constraints to Group Decision-Making. https://doi.org/10.31234/osf.io/6gten 
Williams, Andy E., 2020, d, A Model for General Collective Intelligence. Retrieved from osf.io/preprints/africarxiv/6u984 Williams, Andy E., 2020, e, The Need for General Collective Intelligence in Addressing the Sustainable Development Goals, DOI:10.21203/rs.3.rs-18022/v1. 